\title{
Surfactant controlled switching of water-in-oil wetting behaviour of porous silica films grown at oil-water interfaces ${ }^{\dagger}$
}

\author{
MANISH M KULKARNI, RAJDIP BANDYOPADHYAYA ${ }^{1}$ and ASHUTOSH SHARMA* \\ Department of Chemical Engineering, Indian Institute of Technology Kanpur, Kanpur 208016 , \\ ${ }^{1}$ Present address: Department of Chemical Engineering, Indian Institute of Technology Bombay, \\ Powai, Mumbai 400076 \\ e-mail: rajdip@che.iitb.ac.in; ashutos@iitk.ac.in
}

\begin{abstract}
Selective permeation of oil and water across a porous medium, as in oil recovery operations, depends on the preferential wetting properties of the porous medium. We show a profound influence of surfactants in wetting of porous media and thus demonstrate a new route for the control of water-in-oil wetting of porous substrates by changing the concentration of surfactants in an aqueous sub-phase below the substrate. This strategy is employed to engineer partial reversible wetting transitions on a porous silica film. The film itself is grown and stabilized on a flat, macroscopic interface between an oil phase and an aqueous sub-phase. On increasing the surfactant (CTAB) concentration in the sub-phase, contact angle of a water drop (placed on the oil side of the film) changes from $140^{\circ}$ to $16^{\circ}$ in 25 min by diffusion of the surfactant across the porous film. On further replacement of the sub-phase with pure water, diffusion of the surfactant from the water drop back to the sub-phase was slower, increasing the contact angle in the process from $16^{\circ}$ to $90^{\circ}$ in $2 \mathrm{~h}$. Wettability control by a cationic surfactant (CTAB) was found to be much faster $(6 \mathrm{deg} / \mathrm{min})$ than that offered by an anionic surfactant, SDS $(0.05 \mathrm{deg} / \mathrm{min})$. Switching of the surface wettability due to the surfactant diffusion may have implications in oil-water separation, chemical bed reactors and microfluidic devices.
\end{abstract}

Keywords. Wetting control; porous substrate wetting; porous silica film; surfactant mediated wetting; oil-water contact angle.

\section{Introduction}

Spreading of liquid on a porous solid ${ }^{1}$ and understanding the mechanics of the flow inside porous body are important in adsorbers and reactors with porous packings. ${ }^{2,3}$ The Spreading and penetration of liquids and movement of the three phase (solid-liquidair) contact lines on porous surfaces like paper, fabric, etc have also been studied extensively. ${ }^{4,5}$ Reversible switching of hydrophilic-hydrophobic characteristics of surfaces is desired in many applications. Various methods like electrowetting, ${ }^{6}$ chemical surface modification, ${ }^{7}$ conformational and photo-induced transitions have been reported for dynamic control of surface wetting. Most of these works are concerned with the control of wetting in air-water-surface systems by manipulating the surface properties of a non-porous solid surface. In case of control by electrowetting, the solid surface needs to be electrically active. However, in many practical applications the solid surface properties cannot be readily altered.

\footnotetext{
${ }^{\dagger}$ Dedicated to Prof. C N R Rao on his 75th birthday

*For correspondence
}

In many important applications, such as in oil recovery operations, selective permeation of oil and water across a porous medium depends on the preferential wetting properties of the porous medium. Further, wetting characteristics of porous rocks play a profound role in displacement of oil by water from these surfaces. We show a profound influence of surfactants in wetting of porous media and thus demonstrate a new route for the control of water-inoil wetting of porous substrates by changing the concentration of surfactants in an aqueous sub-phase below the substrate. Towards this end, we employ a model system of a porous silica film, which was grown and stabilized at a flat, macroscopic oil-water interface. We report a new surfactant mediated control of the wetting characteristics of such a porous wall. This is shown by a transition in the contact angle of a water drop placed on the oil-side (like heptane) of the film.

Use of a liquid-liquid interface to grow metallic nanocrystalline films has been described in the seminal works of Rao et $a l^{8,9}$ Similarly, Faget et $a l^{10}$ synthesized mesoporous silica films at an oil-water 
interface. Here, we investigate the barrier properties of the interfacially grown, mesoporous silica films to the movement of oil and water. Clearly, the barrier offered by the mesoporous film depends on its selective wetting by water surrounded by oil. We exploited the porous nature of the silica film to change its wetting properties by diffusion of surfactants across the pores of the film. The advantage of our method is that the solid surface energy need not be modified to change the contact angle. Instead, the interfacial energy of the drop with the surrounding bulk liquid is tuned such that a non-wetting drop of water completely wets the porous solid film. Moreover, this transition is partially reversible. Kumar et $a l^{11}$ have investigated the wetting dynamics of liquid over a porous media and identified various stages of spreading based on the importance of governing forces. However, in our work, the porosity of the solid film plays an important role in a different context in that the change in the interfacial energy of the two liquids is achieved by diffusion of a surfactant to/from the water sub-phase across a solid substrate. This method of controlling the interfacial tension may have potential applications in the related areas of chemical reactors, microfluidic devices such as interfacial tensiometer ${ }^{12,13}$ and oil recovery. ${ }^{14,15}$

\section{Materials and methods}

A silica precursor, like, tetraethoxysilane (TEOS, puriss grade, Fluka chemicals) was dissolved in heptane (HPLC grade, Loba chemicals). This solution was then slowly poured onto a water-ammonia mixture in a way such that the oil-water interface thus formed was free from any bubbles or drops. Typically the molar ratio of TEOS: heptane was kept constant at $1: 7 \cdot 5$, and the aqueous phase had $1 \mathrm{M}$ $\mathrm{NH}_{4} \mathrm{OH}$. For the rest of the paper, we refer to the aqueous phase as the sub-phase. Silica film formation at the heptane-water interface started immediately. The thickness of the film could be controlled by changing the heptane/TEOS molar ratio and the time allowed for growth of the film. Film growth was stopped by removing the top oil (TEOS-heptane) phase, once a silica film of desired thickness was formed. Pure heptane was then poured slowly again with minimum disturbance on the film for further contact angle measurements.

An approximately $5 \mu \mathrm{l}$ water droplet was then introduced in the oil phase, which subsequently settled down on the oil-side surface of the silica film. The droplet was photographed at fixed time intervals of $2 \mathrm{~min}$ up to an overall duration of $10 \mathrm{~h}$ with a horizontal optical microscope at $100 \mathrm{X}$ magnification coupled with a CCD camera capable of capturing images at $25 \mathrm{fps}$. The contact angle $(\theta)$ of the water droplet was calculated by measuring the height $(h)$ and length of the contact line (d) of the droplet. These three parameters are related by the following equation: ${ }^{16}$

$$
\theta=2 \tan ^{-1}\left[\frac{2 h}{d}\right] .
$$

Surface tension of a liquid and liquid-liquid interfacial tension were measured with a tensiometer (Fisher Surface Tensiomat, USA) using a Pt-ring probe. Contact angle and surface tension measurements were repeated thrice to estimate the measurement error.

\section{Results and discussion}

It is well known that hydrolysis and polycondensation of alkoxysilanes in presence of water leads to the formation of silica ${ }^{17}$ However, synthesis of silica films at interfaces is not much explored, apart from the studies of Faget et $a l^{10}$ and Bandyopadhyaya et $a l^{18}$ on mesoporous silica film synthesis at oil-water interface. Recently, we reported synthesis of methyltrimethoxysilane based silica films at the oil-water interface. ${ }^{19}$ It was observed that after drying, these films exhibit Janus hydrophobic-hydrophilic properties across the oil and water side surfaces, respectively. However, in the present study, we use tetraexthoxysilane (TEOS) as the silica precursor and prepared the silica films both without and with ionic surfactants (SDS and CTAB). In presence of a cata-

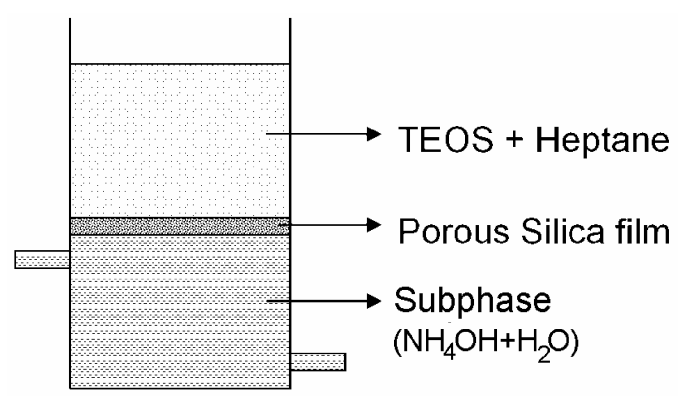

Figure 1. Schematic diagram of experimental set-up for growing silica film at the macroscopic heptane-water interface. 
lyst $\left(\mathrm{NH}_{4} \mathrm{OH}\right)$, TEOS reacts with water to form a random porous network of silica (in the form of a film) at the oil-water interface. We study the wet-

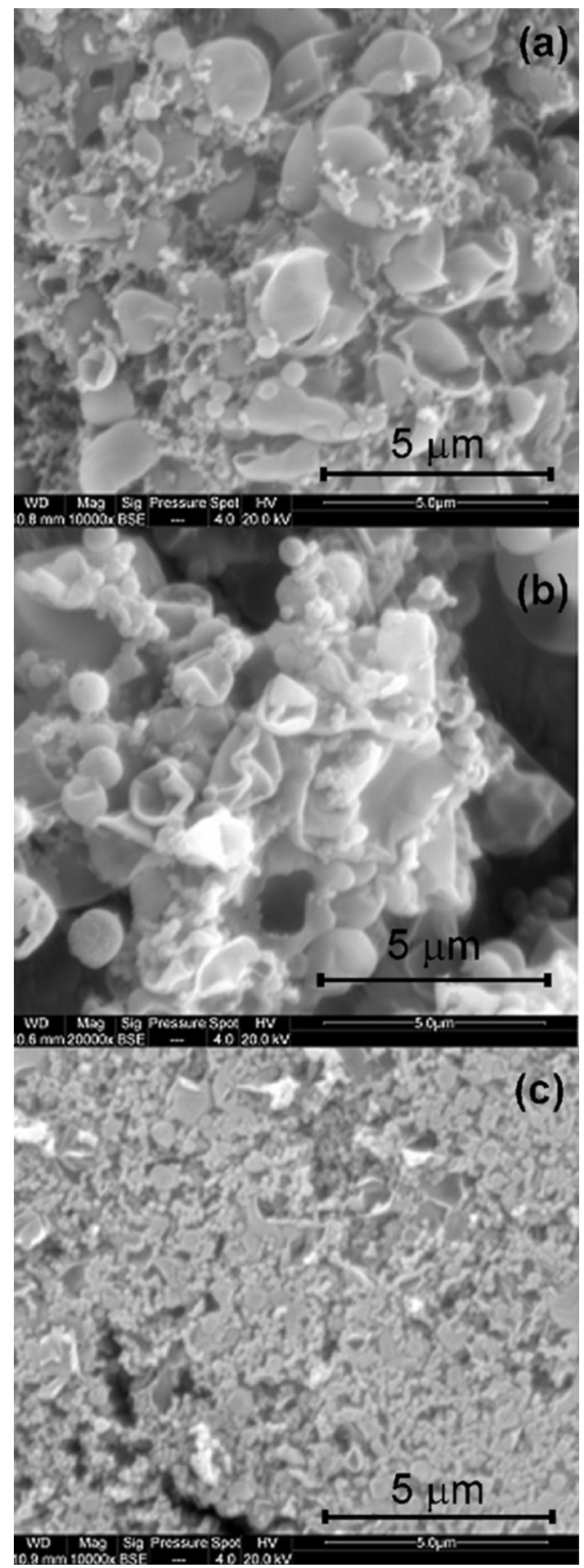

Figure 2. SEM microstructure of silica films synthesized at heptane-water interface using $1 \mathrm{M} \mathrm{NH}_{4} \mathrm{OH}$ subphase: (a) without any surfactant in the sub-phase, (b) 1 CMC CTAB sub-phase and (c) 1 CMC SDS sub-phase (scale bar represents $5 \mu \mathrm{m}$ ). ting properties of such porous films by changing the surfactant concentration of the sub-phase while keeping the film straddled at the oil-water interface. Figure 1 shows a schematic diagram of our experimental set-up.

The silica films synthesized at the oil-water interface are composed of silica clusters connected to each other forming a network structure as shown in figure 2. Figure $2 \mathrm{a}$ shows the microstructure of the silica film prepared using $1 \mathrm{M} \mathrm{NH}_{4} \mathrm{OH}$ in the subphase without any surfactant. Surface of such a film shows a network of hierarchical $0.3-2 \mu \mathrm{m}$ silica clusters with a prominent bimodal size distribution. Macroscopically, the film was relatively smooth. Addition of surfactants to the $1 \mathrm{M}$ ammonia subphase before the formation of silica films affected the growth and microstructural properties of the silica film. SEM micrograph in figure $2 b$ shows that addition of CTAB at around its CMC to the subphase led to the formation of silica films displaying prominently only the large clusters $(\sim 2 \mu \mathrm{m})$. The smaller features visible in figure $2 \mathrm{a}$ are absent. In contrast, the silica films prepared with SDS subphase show much smaller clusters $(\sim 200 \mathrm{~nm})$ and tighter pores (figure $2 \mathrm{c}$ ). Also, in the case of CTAB surfactant sub-phase, the growth rate of the silica film was very fast and 1-2 mm thick films were obtained in $\sim 30 \mathrm{~min}$, whereas very thin films were obtained in the case of SDS even after $24 \mathrm{~h}$. In fact, the silica film prepared using SDS was very brittle and often developed cracks even under the weight of a water drop placed on it. As a result, contact angle measurements could not be performed on these films. Whereas, in the case of CTAB sub-phase, the silica films were very thick, rough and the oil side surface often wrinkled, which obstructed the visibility of water drop-silica film contact line so accurate measurements of the contact angle on these films also could not be performed. In view of the above factors, the films grown without surfactants were found to be most suitable for the contact angle measurements reported below.

Wetting properties of the film were studied by the three phase contact angle measurements in which a silica film straddling at the oil-water interface was used as a substrate. Water completely wets the dried silica film in air although some unreacted hydrophobic $-\mathrm{OC}_{2} \mathrm{H}_{5}$ groups might be present on the film surface ${ }^{17}$ because when exposed to moist air, $\mathrm{Si}-\mathrm{OC}_{2} \mathrm{H}_{5}$ hydrolyse quickly and the film looses its hydrophobicity. ${ }^{20}$ However, it has been observed that in the presence of heptane, a pure water droplet as- 
sumes a nearly spherical shape (contact angle, $\theta \cong 140^{\circ}$ ). Contact angle at the three phase contact line of heptane-water-quartz (which is considered to be almost non-porous) was observed to be $\sim 20^{\circ}$. So it can be concluded from these observations that the higher contact angle for the heptane-water-silica film system is possibly because of: (i) the presence of partially hydrophobic unreacted $-\mathrm{OC}_{2} \mathrm{H}_{5}$ groups from the TEOS precursor on the surface of silica clusters and (ii) the heptane-water interfacial energy being dominant than that of the water drop and the silica surface. The distribution of heptane and water in the pores of the film is complex, but from the higher contact angle of water drop on the oil side surface of the film, it seems that heptane phase dominates in the pores.

Wetting of the silica film by a water droplet can be controlled either by changing the roughness or the chemical property of the film, or, as shown in the present studies, by allowing diffusion of a surfactant across the oil-water drop interface. Since the silica film is porous and there is a concentration gradient, surfactant diffuses from sub-phase to the water drop or vice versa. In this regard, we have studied the effect of two different water soluble surfactants, namely, cetyl trimethyl ammonium bromide (CTAB), and sodium dodecyl sulfate (SDS), which are cationic and anionic, respectively.

Contact angle of the heptane-water-silica film system with pure water as the sub-phase is $140^{\circ}$, as shown in figure $3 \mathrm{a}$. This sub-phase was exchanged with five times the critical micellar concentration (CMC) of CTAB, without disturbing the water droplet or the silica film. As a result, CTAB diffused from the sub-phase to the droplet and the contact angle decreased to $16^{\circ}$ within $25 \mathrm{~min}$ (figure $3 \mathrm{~b}$ ). We found that the rate of change of contact angle was almost constant with time, and once the surfactant concentration in the drop became equal to that in the sub-phase, the contact angle stabilized to $16^{\circ}$.

From table 1 , the experimentally measured value of pure water-heptane interfacial tension is $49 \mathrm{mN} / \mathrm{m}$. With increase in CTAB concentration from 0.1 to 10 times CMC, this interfacial tension decreased from 25 to $1 \mathrm{mN} / \mathrm{m}$. Therefore, with diffusion of CTAB into the droplet, $\theta$ decreased significantly due to the adsorption of CTAB at the oil-water droplet interface. As expected, the rate of change of contact angle increased with increase in sub-phase surfactant concentration. As shown in table 2, this rate increased to $6 \mathrm{deg} / \mathrm{min}$, on increasing CTAB concentration to 5 times $\mathrm{CMC}$, with simultaneous decrease in final equilibrium contact angle. These results show that the change in contact angle is primarily because of diffusion of surfactant from sub-phase to drop, and is a function of surfactant concentration.

To verify that surfactant diffusion takes place from sub-phase to the droplet and vice versa (i.e. a reversible two-way process), next, the sub-phase with CTAB concentration of 5 times CMC was further

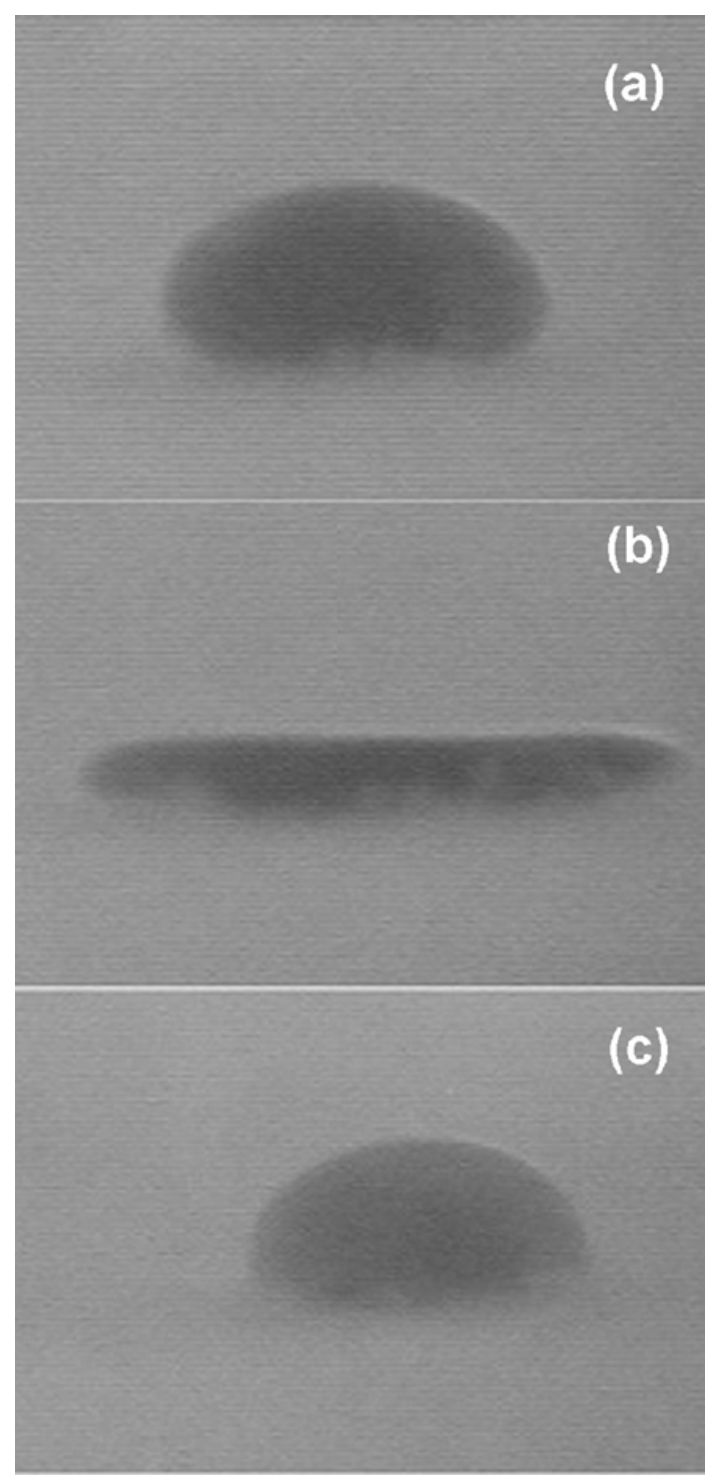

Figure 3. Photographs showing surfactant mediated partial reversible switching of the wetting properties of a porous silica film grown and stabilized at the heptanewater interface. The transitions depicted are hydrophobic to hydrophilic and then back to hydrophobic again. (a) Water drop placed on heptane-side of the silica film with 5 CMC CTAB concentration in the aqueous sub-phase, at the instant $t=0$, with contact angle, $\theta=140^{\circ}$, (b) after $t=25 \mathrm{~min}, \theta=16^{\circ}$ and (c) after replacing the sub-phase back with pure water, $\theta$ increases again to $90^{\circ}$ after $2 \mathrm{~h}$. 
Table 1. Surface and interfacial tensions of various liquids used in the present study. All the surfactant solutions were prepared in aqueous $1 \mathrm{M} \mathrm{NH}_{4} \mathrm{OH}$.

\begin{tabular}{lcc}
\hline Liquid & $\begin{array}{c}\text { Liquid surface tension } \\
(\mathrm{mN} / \mathrm{m})^{\mathrm{a}}\end{array}$ & $\begin{array}{c}\text { Sub-phase/heptane } \\
\text { interfacial tension }(\mathrm{mN} / \mathrm{m})^{\mathrm{a}}\end{array}$ \\
\hline Water & $69( \pm 1)$ & $49( \pm 2)$ \\
Heptane & $21( \pm 2)$ & - \\
$0 \cdot 1$ CMC CTAB & $53( \pm 1)$ & $25( \pm 1)$ \\
1 CMC CTAB & $34( \pm 1)$ & $2( \pm 1)$ \\
5 CMC CTAB & $24( \pm 2)$ & $1( \pm 1)$ \\
$0 \cdot 1$ CMC SDS & $47( \pm 1)$ & $30( \pm 1)$ \\
1 CMC SDS & $34( \pm 1)$ & $5( \pm 2)$ \\
5 CMC SDS & $25( \pm 2)$ & $3( \pm 2)$ \\
\hline
\end{tabular}

${ }^{a}$ Figures in the bracket indicate error estimated from 3 measurements

Table 2. Equilibrium contact angle of three phase contact line of heptane-water-silica film after diffusion of surfactant in to the drop from the sub-phase. The initial contact angle $\left(\theta_{\mathrm{o}}\right)$ was $\sim 140^{\circ}( \pm 5)$.

\begin{tabular}{lccc}
\hline $\begin{array}{l}\text { Type and approx. concentration } \\
\text { of the surfactant in the } \\
1 \mathrm{M} \mathrm{NH} \mathrm{NH}_{4} \mathrm{OHb} \text {-phase }\end{array}$ & $\begin{array}{c}\text { Equilibrium } \\
\text { contact angle }\end{array}$ & $\begin{array}{c}\text { Time interval for the } \\
\text { equilibrated contact angle (min) }\end{array}$ & $\begin{array}{c}\text { Rate of change of contact } \\
\text { angle degrees/min (approx.) }\end{array}$ \\
\hline 1 CMC CTAB & $47^{\circ}( \pm 4)$ & 25 & 4 \\
5 CMC CTAB & $16^{\circ}( \pm 5)$ & 25 & 6 \\
1 CMC SDS & $128^{\circ}( \pm 3)$ & 600 & $0 \cdot 025$ \\
5 CMC SDS & $110^{\circ}( \pm 5)$ & 600 & $0 \cdot 05$ \\
\hline
\end{tabular}

${ }^{\mathrm{a}}$ Figures in the bracket indicate error estimated from 3 independent measurements

exchanged with pure water. Figure $3 \mathrm{c}$ shows the water droplet in equilibrium, after the surfactant diffusion from water drop back to the sub-phase was complete. This process took $2 \mathrm{~h}$ and the contact angle increased to $90^{\circ}$, indicating the process is partially reversible. This reverse diffusion of surfactant was slower, with the rate of change of contact angle being $0.75 \mathrm{deg} / \mathrm{min}$. This may be due to the smaller concentration gradient between a $15 \mu \mathrm{l}$ CTAB rich droplet and the large pool $(\sim 50 \mathrm{ml})$ of pure water, in this case, as compared to the pure water drop-CTAB sub-phase system discussed earlier. However, for a fresh drop of 1 CMC CTAB solution placed on the silica film with pure water as sub-phase, contact angle increased from $10^{\circ}$ to $140^{\circ}$ in $80 \mathrm{~min}$ (rate of change of contact angle being $\sim 1.5 \mathrm{deg} / \mathrm{min}$ ). This indicates that the surfactant adsorbs on the silica film. When the sub-phase is rich in surfactant, it adsorbs on the silica film as well as diffuses to the drop. After replacing the surfactant rich sub-phase with pure water, the contact angle increases slowly, which means the surfactant concentration in the drop and on the film surface is almost similar and the surfactant desorbs from the film surface very slowly leading to a slow diffusion of surfactant away from the drop into the sub-phase. However, when there was no surfactant adsorbed initially on the film, the diffusion of surfactant from the drop to the film and the sub-phase are very rapid.

To study the effect of surfactant head group charge on its diffusion through the silica film, contact angle of water droplet was also measured as a function of anionic surfactant sodium dodecyl sulfate's (SDS) concentration in the sub-phase. Although the interfacial tension for heptane-surfactant solution is almost independent of the type of surfactant, the change in contact angle was very slow in case of a sub-phase with SDS. As seen from table 2, the rate of change of contact angle for SDS was slower by two orders of magnitude, compared with a solution with similar CTAB concentration. To study SDS diffusion in the reverse direction, a drop containing SDS solution was placed on the silica film with water as subphase. For a droplet with $1 \mathrm{CMC}$ SDS, the rate of change of contact angle was very slow $(\sim 0.03$ $\mathrm{deg} / \mathrm{min})$, as the contact angle increased from $30^{\circ}$ to only $32^{\circ}$ in $60 \mathrm{~min}$.

Both CTAB and SDS have similar size of molecules and the radius of micelles $(\sim 3 \mathrm{~nm})$ formed by these surfactants (above CMC) is much smaller than 
the pore sizes $(>100 \mathrm{~nm})$ of the silica films prepared here. Hence the rate of diffusion through the silica film should be similar for both CTAB and SDS. Therefore, one of the possible reasons for selective diffusion could be the fact that silica has terminal $-\mathrm{OH}$ groups on its internal pore surface. ${ }^{14}$ The anionic head group of SDS may interact with silica surface and get adsorbed on it strongly as compared to CTAB. This can be further facilitated by the very high surface area of these films due to the threedimensional porous network of the silica clusters, hindering further diffusion of SDS molecules through the porous film. The contact angle switching behaviour shown here was persistent for at least three different cycles of sub-phase and droplet replacements, which indicates that the surfactant adsorbs strongly on the silica surface. Although the mechanisms of the difference in switching of contact angle after the CTAB and SDS adsorption need to be investigated further, these results indicate that the silica films grown here can be used for the control of wetting characteristics by surfactant diffusion.

\section{Conclusion}

A method to control wetting properties of a porous silica film grown and stabilized at a flat, macroscopic heptane-water interface (with water as the subphase) has been described. On changing the concentration of the dissolved surfactant in the aqueous sub-phase, the wetting characteristics of the porous film could be altered in a partial reversible manner. Such a switching of wetting characteristics takes place due to diffusion of the surfactant; it can be either from the aqueous sub-phase to a water drop placed on the heptane side of the film, or in the reverse direction, depending on the concentration gradient of the surfactant across the film. For a pure water drop placed on the silica film and the aqueous sub-phase replaced by five times the critical micellar concentration (CMC) of a cationic surfactant (CTAB), the contact angle decreased from $140^{\circ}$ to $16^{\circ}$ in $25 \mathrm{~min}$. This is because of diffusion of CTAB from sub-phase to the water drop and its adsorption at the water drop-oil interface. For surfactant diffusion in the reverse direction (i.e. from the water drop to the sub-phase), the two cases examined by changing the drop history gave similar reversibility in wetting transition: (i) if the surfactant sub-phase was replaced back by pure water, the contact angle increased from $16^{\circ}$ to $90^{\circ}$ in $2 \mathrm{~h}$, and (ii) for a fresh drop containing
$1 \mathrm{CMC}$ aqueous $\mathrm{CTAB}$ solution placed on the silica film, with pure water as the sub-phase, contact angle increased from 10 to $140^{\circ}$ in $80 \mathrm{~min}$. This discrepancy in the rate of change of contact angle indicates that the surfactant diffusion from drop to sub-phase is slow when there is large amount surfactant adsorbed on the silica film initially. We also found that diffusion rate of cationic CTAB was much faster compared to the anionic SDS surfactant, indicating selectivity of the porous silica film towards diffusion of CTAB. Thus, the porous silica film can possibly be used as a membrane for selective surfactant diffusion. The results presented have implications in oil-water separation and microfluidic devices.

\section{Acknowledgements}

AS acknowledges the support of the Department of Science and Technology (DST) through its grants to the Unit on Nanosciences at Indian Institute of Technology (IIT), Kanpur and an IRHPA project. It is a pleasure to be a part of the Festschrift honouring Professor C N R Rao whose life and works have been a constant source of inspiration to us.

\section{References}

1. Starov V M, Zhdanov S A, Kosvintsev S R, Sobolev V D and Velarde M G 2003 Adv. Coll. Inter. Sci. 104 123

2. Maiti R N, Khanna R, Sen P K and Nigam K D P 2004 Chem. Engg. Sci. 592817

3. Khanna R and Nigam K D P 2002 Chem. Engg. Sci. 573401

4. Danino D and Marmur A 1994 J. Colloid Interface Sci. 166245

5. Pezron I, Bourgain G and Quere D 1995 J. Colloid Interface Sci. 173319

6. Verheijen H J J and Prins M W J 1999 Langmuir 15 6616

7. Xia F, Feng L, Wang S, Sun T, Song W, Jiang W and Jiang L 2006 Adv. Mater. 18432

8. Rao C N R, Kulkarni G U, Thomas P J, Agrawal V V and Saravanan P 2003 J. Phys. Chem. B107 7391

9. Rao C N R, Kulkarni G U, Agrawal V V, Gautam U $\mathrm{K}$, Ghosh M and Tumkurkar U $2005 \mathrm{~J}$. Colloid Interface Sci. 289305

10. Faget L, Berman A and Regev O 2001 Thin Solid Films 3866

11. Kumar S M and Deshpande A P 2006 Colloids and Surfaces A Physicochem. Eng. Aspects 277157

12. Cabral J T and Hudson S D 2006 Lab on a Chip 6427

13. Geerken M J, Lammertink R G H and Wessling $\mathrm{M}$ 2007 Colloids and Surfaces A: Physicochem. Eng. Aspects 292224 
14. Touhami Y, Hornof V and Neale G H 1998 Colloids and Surfaces A: Physicochem. Eng. Aspects 13261

15. Zhao Z, Bi C, Qiao W, Li Z and Cheng L 2007 Colloids and Surfaces A: Physicochem. Eng. Aspects 294 191

16. Bikerman J J 1958 Surface chemistry: Theory and applications (New York: Academic Press) 2nd edn, p. 343
17. Brinker C J and Scherer G W 1990 Sol-gel Science (San Diego: Academic Press) p 523

18. Bandyopadhyaya R, Nativ-Roth E, Yerushalmi-Rozen $\mathrm{R}$ and Regev O 2003 Chem. Mater. 153619

19. Kulkarni M M, Bandyopadhyaya R and Sharma A 2008 J. Mater. Chem. 181021

20. Schwertfeger F, Zimmermann A and Krempel $\mathrm{H}$ 2001 U.S. Patent No. 6280744 